\title{
Avaliação da Escalabilidade de Aplicações de Alto Desempenho em Nuvem Pública e Privada
}

\author{
Ariel Lauber de P. Silva ${ }^{1}$, Thiago B. de Oliveira ${ }^{1}$ \\ ${ }^{1}$ Bacharelado em Ciências da Computação - Universidade Federal de Goiás (UFG) \\ Regional Jataí - Campus Cidade Universitária - BR 364, KM 195, 3800 \\ CEP: 75801-615 - Jataí - GO - Brasil \\ ariellauberegmail.com, thborgeseufg.br
}

\begin{abstract}
The high performance computing application analysis $(H P C)$, has recently been facilitated by the use of cloud platforms. However, the performance of HPC applications relies heavily on the support I/O platform, mainly the communication network between the VMs ${ }^{1}$. In this study, the application performance of the NPB-NAS Parallel Benchmark suite and DGEO suite, which uses processing spatial queries was compared in a physical cluster, a private cloud, XenServer, a public cloud, Microsoft Azure. The tests demonstrate one speed up to 3.0 times lower in the public cloud for limited by CPU applications, and a runtime up to 3 times greater than the physical cluster. The speed-up between cloud platforms tested here was analyzed that it is not only affected by the extra layer of virtualization, but also by other factors platform cloud as VMs neighbors.
\end{abstract}

Resumo. A avaliação de desempenho de aplicações de computação de alto desempenho (HPC) foi recentemente facilitada pelo uso de plataformas em nuvem. No entanto, o desempenho de aplicativos HPC depende fortemente do suporte de I/O da plataforma, principalmente da rede de comunicação entre as $V M s$. Neste estudo apresentamos o resultado de uma avaliação de escalabilidade de aplicações HPC em cluster físico, nuvem privada usando XenServer e em nuvem pública, Microsoft Azure. Os testes demostram um speed-up até 3,0 vezes menor na nuvem pública para aplicações limitadas por CPU, e um tempo de execução até 3 vezes maior em relação ao cluster físico. Os resultados indicam que, apesar de conveniente, testar a escalabilidade de aplicações distribuídas em plataformas virtuais (nuvem pública ou privada) pode levar a interpretações incorretas ou imprecisas a respeito da escalabilidade de alguns tipos de aplicações distribuídas.

\section{Introdução}

O aumento recente da disponibilidade de plataformas de nuvem, principalmente fornecedores de infraestrutura como serviço (IaaS), tem causado uma migração da análise de aplicações de computação de alto desempenho (HPC - High Performance Computing). Aplicações e serviços que antes eram testadas exclusivamente em clusters ou servidores nos centros de computação nas universidades ou em pequenas e médias empresas, são hoje testadas e hospedadas em clusters virtuais na nuvem. Os subsídios concedidos pelos

\footnotetext{
${ }^{1}$ Virtual Machine (VM).
} 
fornecedores de infra-estrutura para projetos de pesquisa nas universidades também tem contribuído neste sentido ${ }^{2}$.

O uso de plataformas de computação em nuvem é vantajoso devido a três fatores principais: $i$ ) a facilidade de configurar e manter clusters para execução de aplicações HPC, $i$ i) a inexistência do custo inicial para adquirir a infraestrutura necessária e, iii) a elasticidade, que proporciona o aumento da infraestrutura sob demanda, fato primordial para o teste de aplicações distribuídas.

Apesar dos avanços das tecnologias de compartilhamento de I/O em VMs, como Intel VT-X [Abramson et al. 2014] e AMD-V IOMMU (AMD) [AMD 2011], alguns aspectos da virtualização ainda causam preocupação atualmente. Um deles é a interferência de VMs vizinhas, alocadas na mesma máquina física. Esta estratégia é empregada pelos provedores de computação em nuvem, com objetivo de reduzir custos e o consumo de recursos naturais [Ferreira et al. 2014]. O isolamento entre as VMs, ainda hoje, é um problema reportado por alguns artigos como [Mei et al. 2013, Tudoran et al. 2012, Barker e Shenoy 2010, Hill et al. 2010]. Este fato, se não observado adequadamente, compromete a repetibilidade dos experimentos, conforme discutido por [Bakhshayeshi, Akbari e Javan 2014].

Neste trabalho foi realizada uma avaliação de escalabilidade (speed-up) e de tempo de processamento em plataforma de nuvem pública Azure ${ }^{3}$, nuvem privada XenServer ${ }^{4}$, e em uma cluster físico, sem virtualização. Foram usadas duas suítes de aplicações para obter valores representativos de speed-up: a suíte NPB-NAS Parallel Benchmark [Bailey et al. 1991] e o banco de dados espacial experimental DGEO [Oliveira, Costa e Rodrigues 2015].

Este artigo expande nosso trabalho anterior, publicado em [Oliveira et al. 2015], incluindo a avaliação das mesmas aplicações em uma nuvem privada, a fim de esclarecer se o resultado anterior, em relação ao menor desempenho das aplicações na nuvem pública, se deve à concorrência de VMs vizinhas ou ao overhead da tecnologia de virtualização empregada pelo provedor de serviços.

As principais contribuições deste trabalho são:

- Avaliação comparativa de aplicações de HPC entre cluster físico, nuvem pública e privada, afim de identificar possíveis diferenças de tempo de execução e valores de speed-up;

- Avaliação de aplicações com níveis de speed-up diferenciados e representativos;

- Avaliação do comportamento de aplicações CPU-bound e Network-bound; e

- Identificação de comportamentos que interferem na escalabilidade, tanto na nuvem pública, privada e no cluster físico.

\section{Trabalhos Correlatos}

Apesar do grande esforço em medir o desempenho de plataformas de computação em nuvem, fato que pode ser notado devido ao grande volume de publicações recentes que

\footnotetext{
${ }^{2} \mathrm{~A}$ Microsoft e a Amazon possuem programas universitários de apoio à pesquisa em suas respectivas plataformas de computação em Nuvem: Azure e Elastic Compute Cloud (EC2).

${ }^{3}$ https://azure.microsoft.com/en-us/

${ }^{4}$ http://xenserver.org/
} 
fazem avaliações nos ambientes em nuvem [Sivathanu e Dong 2013], a quantidade de variáveis que interferem na comparação é muito grande e isso dificulta uma avaliação completa das plataformas. Como é afirmado por [Hill et al. 2010], não há uma taxonomia de avaliação amplamente aceita e muitos trabalhos confundem os termos ou realizam experimentos inconsistentes para avaliar determinadas características.

A maioria dos trabalhos analisam comparativamente as implementações de VMM existentes, como [Walters et al. 2008, Huber et al. 2011, Bakhshayeshi, Akbari e Javan 2014]. Nota-se uma instabilidade em relação às conclusões, devido a constates evolução das tecnologias de virtualização e da recente disponibilização de hardware com suporte à virtualização (Intel VT-D e AMD-V). Alguns trabalhos avaliam o desempenho de diferentes tecnologias de virtualização (Paravirtualização, Virtualização Completa, e outras), como é o caso do trabalho de [Younge et al. 2011]. Atualmente, os principais VMMs existentes implementam somente a tecnologia de Paravirtualização ou Virtualização Completa.

O estudo de [Ferreira et al. 2014] conclui que o aumento da quantidade de VM por máquina física é o fator mais relevante na redução do desempenho de VMs vizinhas, devido à maior concorrência por recursos na máquina física. A falta de controle da vizinhança por parte do usuário da plataforma e a falta de isolamento do VMM, comprometem a repetibilidade dos experimentos, conforme é discutido em [Tudoran et al. 2012, Mei et al. 2013, Bakhshayeshi, Akbari e Javan 2014].

Para demostra a variação de desempenho devido a este compartilhamento, [Tudoran et al. 2012] avaliou comparativamente uma nuvem privada, Nimbus e a nuvem pública, Azure. A conclusão do estudo em relação ao desempenho foi que há uma variabilidade considerável numa janela de tempo de uma semana. O desvio padrão do percentual de variação relatados foram de 24,2\% para a nuvem Nimbus contra $52,3 \%$ para as instâncias ExtraLarge ${ }^{5}$ e $120,7 \%$ para Small ${ }^{6}$ na nuvem Azure. Outros resultados reafirmam a falta de isolamento entre as VMs vizinhas como os apresentados em [Mei et al. 2013, Barker e Shenoy 2010, Hill et al. 2010].

Poucos estudos avaliam a consequência destas conclusões na escalabilidade de aplicações distribuídas em clusters de VMs. O trabalho mais relevante encontrado foi [Expósito et al. 2013]. Nele, aplicações de HPC selecionados da suíte NPB-NAS Parallel Benchmark foram avaliadas na plataforma EC2 da Amazon. No entanto não foi realizado um estudo comparativo entre as plataformas em nuvem pública, privada e cluster físico.

Neste estudo, expandimos os resultados publicados em [Oliveira et al. 2015], que comparou os valores de speed-up de aplicações selecionadas de HPC entre um cluster virtual em nuvem pública e um cluster físico. Os resultados reportados, apesar de conclusivos em relação a escalabilidade e tempo de execução, não identificaram se o overhead encontrado na plataforma pública se devia ao uso de VMs vizinhas ou à tecnologia de virtualização utilizada na plataforma pública. Neste trabalho, realizamos a avaliação das mesmas aplicações em uma nuvem privada, usando XenServer, tecnologia diferente da usada na nuvem pública, e comparamos os resultados entre as três plataformas.

\footnotetext{
${ }^{5}$ ExtraLarge: 8 vCPUs e 14 GB de RAM, e com interface de rede exclusiva.

${ }^{6}$ Small: Instância com 1 vCPU e 1,75 GB de RAM, e interface de rede compartilhada.
} 


\section{Metodologia de Avaliação}

Para os experimentos, as aplicações foram selecionados, observando esses fatores:

1. O speed-up da aplicação, de forma a ter valores representativos, tanto de speed-up próximo a nulo quanto próximo a linear;

2. A diversidade da aplicação em relação ao recurso computacional, tais como: rede ou CPU, e;

3. Se a aplicação explora múltiplos níveis de paralelismo, ou seja, além do paralelismo inerente a distribuição, a aplicação também realiza processamento paralelo multithread, preparada para o uso de máquinas multicore.

Para a aferição dos valores de speed-up, clusters de tamanhos variados foram configurados, com o objetivo foi aumentar a taxa de utilização da interface de rede, pois este é o principal limitador de speed-up em sistemas distribuídos. Ao testarmos um cluster com 8 CPUs ou 8 vCPUs, por exemplo, preferiu-se utilizar 8 máquinas com 1 núcleo habilitado em cada máquina, de forma que as 8 máquinas efetuem comunicação entre si pela interface de rede.

\section{Aplicações Executadas nos Experimentos}

Um conjunto de aplicações da suíte NPB-NAS foram usadas nos experimentos. As aplicações, também chamadas kernels, são escritas em linguagem C e Fortran, utilizando a especificação de comunicação distribuída MPI (Message Passing Interface). A implementação de MPI utilizada foi o OpenMPI ${ }^{7}$. As aplicações escolhidas são as que mais usam banda de rede durante sua execução, conforme indicado em [Bailey et al. 1991, Expósito et al. 2013]. São elas:

1. I $S_{C}$ : Realiza ordenação de números inteiros. Usa all-reduce e comunicação ponto-a-ponto na inicialização;

2. $M G_{C}$ : Mult-Grid numa sequência de de Meshes, comunicação;

3. $C G_{C}$ : Resolve um sistema linear com o método do Gradiente Conjugado;

4. $F T_{C}$ : Transformada Rápida de Fourier 3D. Uso extensivo de primitivas all-to-all que sobrecarregam a rede.

Embora a suíte NPB-NAS seja referência para mensurar o desempenho de máquinas paralelas (com memória compartilhada ou distribuída), os autores afirmaram que usaram instâncias simplificadas de programas reais, que possuem casos melhores de balanceamento de carga [Bailey et al. 1991]. Por isso, a aplicação DGEO [Oliveira, Costa e Rodrigues 2015] foi utilizada nos experimentos. Esta aplicação processa consultas reais de junção espacial, de forma paralela e distribuída. Foi desenvolvida em um projeto de pesquisa em nossa universidade, utilizando linguagem $\mathrm{C}$ e Go, com threads nativas para explorar múltiplos níveis de paralelismo e comunicação distribuída através de protocolos próprios, usando serialização Gob, sobre sockets TCP.

No DGEO, três consultas espaciais foram empregadas nos testes $\left(Q_{a}, Q_{b}\right.$ e $\left.Q_{c}\right)$. Cada consulta possui um speed-up particular, devido às dependências existentes entre os dados espaciais. As consultas foram escolhidas de forma a representar o comportamento de aplicações pouco escaláveis, $\frac{1}{4}$ speed-up escaláveis e $\frac{1}{2}$ speed-up escaláveis.

\footnotetext{
${ }^{7}$ www.open-mpi.org
} 
A cada teste executado, todos os processos de SO referentes às aplicações foram encerrados e reiniciados, com o objetivo de não reaproveitar caches da aplicação, do SO ou do hardware. Nenhuma das aplicações utilizam dados armazenados em disco local na inicialização, e não foi necessário, portanto, limpar a cache de disco do SO.

Os tempos de inicialização das aplicações foram descartados, e somente o tempo de processamento efetivo do algoritmo distribuído foi considerado. Cada uma das aplicações foi executada cinco vezes ${ }^{8}$. Os valores apresentados na seção de resultados foram calculados ignorando o maior e o menor tempo encontrados, e uma média dos outros três valores foi calculada. Nos testes realizados na nuvem pública, as medições foram comparadas em dias e momentos diferentes, para garantir que os tempos não sofreram interferência momentâneas da plataforma.

\section{Configuração dos Clusters utilizados}

Nos experimentos realizados na nuvem pública, privada e no cluster físico, foi usado um total de 16 máquinas em cada. O cluster físico utilizou um conjunto de máquinas do Laboratório de Redes de Computadores (LARC), do Curso de Ciência da Computação da Regional Jataí, da Universidade Federal de Goiás. O cluster virtual na nuvem pública foi configurado na plataforma Azure, da Microsoft, com instâncias de VMs de tamanho A1 a A3. A tabela 1 detalha o hardware completo das máquinas do cluster físico e virtuais da nuvem pública.

Tabela 1. Detalhes do Hardware dos clusters utilizados.

\begin{tabular}{lll}
\hline Item & Cluster Físico & Cluster Virtual (Azure) \\
\hline Quantidade de Máquinas & 16 & 16 \\
CPU & Intel Core i5 $33303 \mathrm{GHz}$ & Intel Xeon E5-2660 $2.2 \mathrm{GHz}$ \\
Núcleos & 4 & $8^{a}$ \\
Cache & $6 \mathrm{MB}$ & $20 \mathrm{MB}$ \\
RAM & DDR3 SDRAM 4 GB $1.333 \mathrm{MHz}$ & Não documentado \\
Rede & $1 \mathrm{Gbps}$ & $1 \mathrm{Gbps}$ \\
Sistema Operacional & XenServer 6.5 & Ubuntu Server 14.4 LTS \\
Switch & D-Link DGS-1210-28P Gigabit & Não documentado \\
& &
\end{tabular}

${ }^{a} \mathrm{O}$ processador Xeon E5-2660 possui 8 núcleos. Neste experimento, duas VMs podem ser vizinhas no mesmo chassi.

${ }^{b} \mathrm{O}$ tamanho é particular da instância e foi limitado de acordo com cada instância escolhida, para refletir a mesma quantidade da máquina física.

${ }^{c}$ Como é comum entre os provedores de plataforma de nuvem, a descrição completa dos equipamentos físicos não é fornecida.

A nuvem privada usou como hypervisor o XenServer 6.5. Em cada máquina física da nuvem privada, foi criada uma VM com SO Ubuntu Server 14.04 LTS 64 bits.

O cluster físico foi configurado com SO Ubuntu Server 14.04 LTS 64 bits. Para todos os testes realizados localmente nas dependências do LARC (nuvem privada e cluster físico) foi criado um servidor DHPC local pra prover o isolamento da rede das máquinas com a rede da Universidade.

A quantidade de núcleos de processamento do cluster físico e nuvem pública variou de 1 a 64 núcleos: de 1 máquina, com apenas um núcleo habilitado, até 16 máquinas

\footnotetext{
${ }^{8}$ Um número maior de execuções não foi necessário devido ao baixo desvio padrão das amostras.
} 
com 4 núcleos habilitados em cada máquina. Os tamanhos de instância foram escolhidos de forma a usar ao máximo a interface de rede disponível. A Tabela 2 detalha a composição de cada instância na nuvem pública.

Para limitar a quantidade de núcleos nas máquinas do clusters físico, e na nuvem privada XenServer, para os experimentos com 1 ou 2 núcleos de CPUs, os núcleos foram desabilitados através do comando específico do GNU/Linux (echo $0>$ /sys/devices/system/cpu/cpuX/online).

Tabela 2. Instâncias utilizadas nos experimentos.
\begin{tabular}{c|c|c|c}
\hline Núcleos & Máquinas & Núcleos p/ Maq & Instância Azure \\
\hline 1 & 1 & 1 & $\mathrm{~A} 1$ \\
2 & 2 & 1 & $\mathrm{~A} 1$ \\
4 & 4 & 1 & $\mathrm{~A} 1$ \\
8 & 8 & 1 & $\mathrm{~A} 1$ \\
16 & 16 & 1 & $\mathrm{~A} 1$ \\
32 & 16 & 2 & $\mathrm{~A} 2$ \\
64 & 16 & 4 & $\mathrm{~A} 3$ \\
\hline
\end{tabular}

Na nuvem pública Azure, as VMs foram desligadas e redimensionadas com a quantidade de vCPUs indicada em cada instância. Como no ambiente Azure a quantidade de RAM aumenta à medida que a quantidade de vCPUs aumenta, para prover o mesmo comportamento em relação à disponibilidade de RAM, foi criado um aplicativo que reserva a quantidade de RAM excedente (em relação às máquinas físicas) e evita paginação, através da chamada de sistema 'mlock'. Este aplicativo foi executado antes de cada teste com tamanho diferente de instância.

\section{Avaliação de Desempenho}

Esta seção avalia os resultados obtidos, conforme a metodologia descrita dos cenários na seção anterior.

\section{Tempo de Execução das Aplicações DGEO}

Mesmo com a diferença de entre o hardware do cluster físico e da nuvem pública, ou seja, $i$ ) apesar do maior clock de CPU no cluster físico (3 GHz x $2.2 \mathrm{GHz}$ ) e ii) uso de processadores específicos para servidores na nuvem pública, o Intel Xeon, com memória cache de CPU maior (20 MB x $6 \mathrm{MB}$ ), os experimentos identificam, na média entre todas as execuções das aplicações classificadas com $C P U$-bound, que o tempo de execução em ambiente Azure (TA) é até três vezes maior, quando comparado com o cluster físico (TF), assim como apresenta a Figura 1.

Somente a diferença percentual de clock que é de 33,6\% não pode justificar essa variação de até $\approx 300 \%$ mais rápido em ambiente físico. Como essas aplicações $C P U$ bound não são intensivas de rede, disco e nem RAM, a diferença se deve ao próprio ambiente compartilhado de nuvem pública, assim reafirmando os resultados discutidos nos trabalhos de [Sivathanu e Dong 2013], [Barker e Shenoy 2010] e [Hill et al. 2010], ou seja, há uma perda de desempenho no ambiente em nuvem, em relação aos tempos de execução, mesmo com uma maior qualidade dos processadores utilizados.

A Figura 2 apresenta a comparação entre os ambientes virtualizados: Azure e XenServer. Observando a figura é possível notar que o tempo de execução das aplicações 


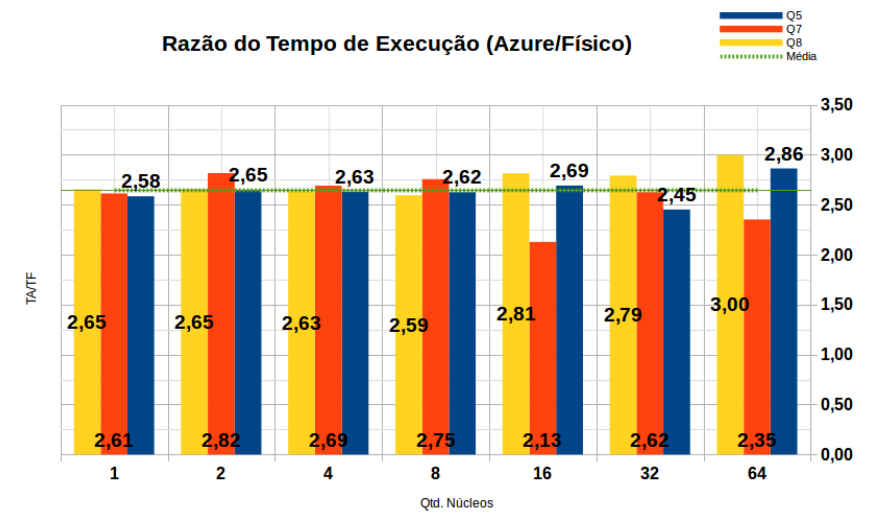

Figura 1. Tempo de execução do Azure e o cluster físico.

CPU-bound no XenServer é menor em relação ao Azure, sendo que essa variação máxima é de 2,82 vezes maior no tempo de processamento no Azure.

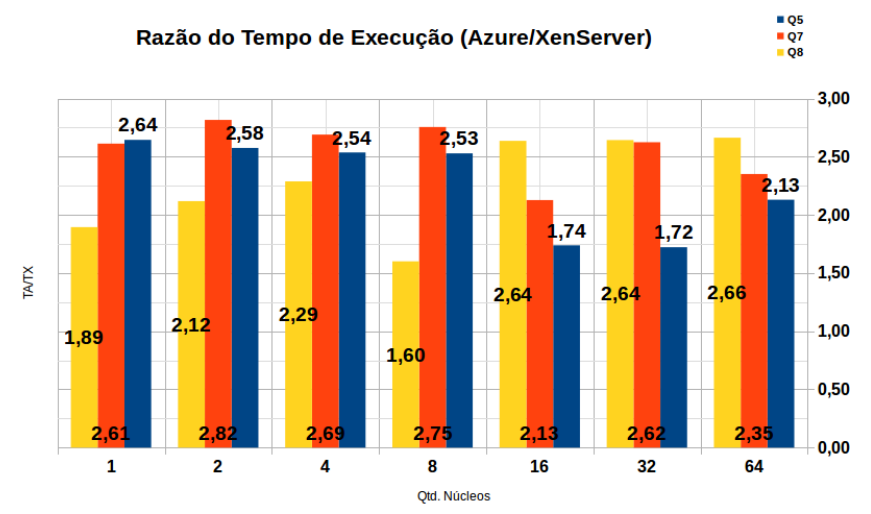

Figura 2. Tempo de execução entre o Azure e o XenServer.

A Figura 3 apresenta a relação de tempo entre o XenServer e o cluster Físico. Constata-se uma maior proximidade entre o tempo de execução nos dois ambiente, comparando com (Azure $x$ Físico). A proporção atinge no máximo 1,62 vezes, mas na média é de 0,21 vezes. Conclui-se, portanto, que para as aplicações $C P U$-bound utilizadas nestes experimentos, a camada de virtualização interferiu substancialmente no tempo de execução das aplicações e o maior overhead foi no ambiente Azure.

\section{Tempo de Execução das Aplicações NPB-NAS}

Analisamos também as aplicações da suíte NPB-NAS. Na Figura 4 temos a relação de tempo gasto em ambiente Azure em relação ao físico. Com a análise podemos averiguar que de modo geral, o físico foi no máximo 3,52 vezes mais rápido nos processamentos das aplicações de benchmarks que a plataforma em nuvem pública Azure, resultado similar aos testes com a aplicação DGEO.

Um comportamento importante na Figura 4 é em relação a quantidade de núcleos. Conforme aumenta-se os núcleos de processamento, ou seja, 1, 2, 4, 8, 16, 32 e 64, a razão do tempo de processamento entre a nuvem pública e o cluster físico vai acompanhando a curva da quantidade de núcleos, na segunda escala do eixo Y. Esse comportamento pode 


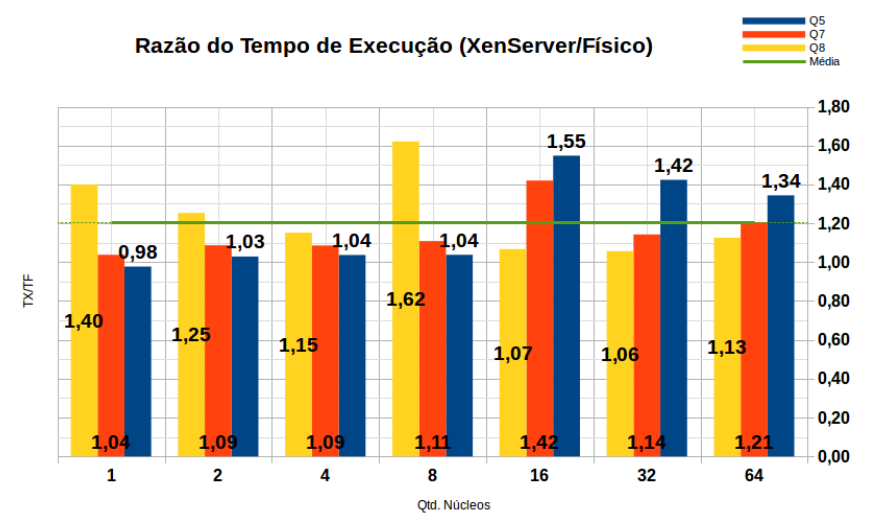

Figura 3. Tempo de execução entre o XenServer e Físico.

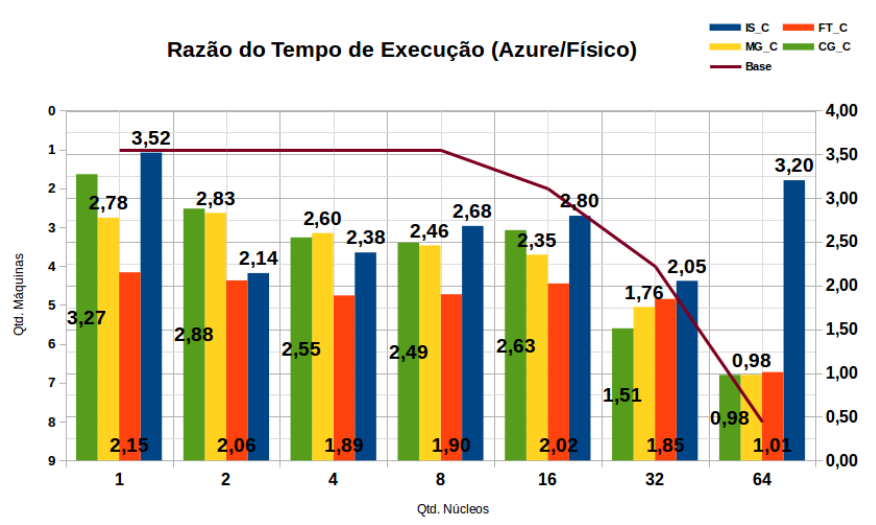

Figura 4. Tempo entre a plataforma Azure e cluster físico.

ser justificado devido ao uso de VMs vizinhas, ou seja, mais de uma máquina virtual em funcionamento no mesmo chassi: enquanto as máquinas virtuais estão no mesmo chassi (cada máquina física na plataforma Azure possui oito núcleos), a linha permanece estável. Porém, de 8 núcleos em diante a linha começa a acompanhar a razão entre as plataformas. Este comportamento fez com que toda a comunicação entre essas máquinas seja realizada dentro do hypervisor, sem comunicação real pela interface de rede. Esse experimento apresenta mais um aspecto importante a ser observado na comparação de escalabilidade em ambiente virtual público, pois o pesquisador não tem controle sobre este aspecto através da interface do provedor.

A Figura 5 compara o tempo do Azure e do XenServer. Verificamos que conforme aumenta-se a quantidade de núcleos a razão entre as duas plataforma diminui, ou seja, os tempos são mais similares entre nuvem pública e nuvem privada.

O comportamento das aplicações da suíte NPB-NAS, que obtiveram valores gradativamente menores em ambiente de pública quando comparados com a nuvem privada, é explicado em duas formas: $i$ ) pela própria característica das aplicações de serem network-bound e ii) na nuvem termos uma maior oferta de banda de rede, ou seja, o uso das instâncias A2 e A3 na nuvem pública Azure, que possuem maior quantidade de banda de rede disponível, e segundo o fato de que em ambiente Azure os servidores físicos possam estar utilizando de VMs vizinhas, fato já reportado por [Sivathanu e Dong 2013] e [Tudoran et al. 2012]. Com o uso de VMs vizinhas, toda a comunicação que seria feita 


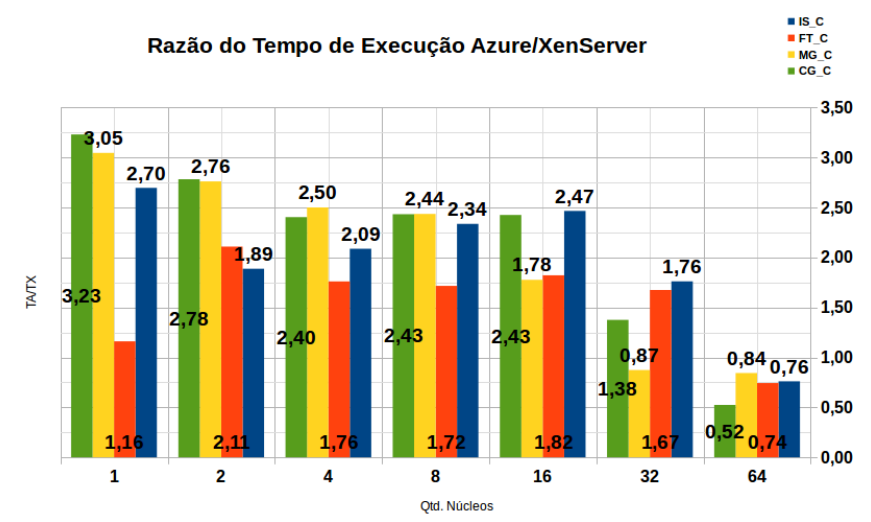

Figura 5. Tempo do Azure e o XenServer.

via rede, passa a ser feita internamente entre as VMs, com isso a taxa de comunicação é superior se comparada com máquinas físicas.

\section{Escalabilidade das Aplicações}

Nesta seção apresentamos a escalabilidade das aplicações das suítes NPB-NAS e DGEO. Durante os experimentos foi constatado três comportamentos distintos das aplicações em relação ao valor do speed-up: i) a aplicação analisada se comporta de forma similar em todos os ambientes, $i i)$ o valor do speed-up é significativamente maior em ambiente físico e iii) o speed-up é significativamente maior em ambiente em nuvem.

A Figura 6 apresenta o resultado de escalabilidade nos três ambientes. Para as aplicações da suíte DGEO, o comportamento escalável entre as aplicações nos três ambientes ocorreu conforme esperado, sendo a ordem decrescente de escalabilidade temos o Q5, Q7 e Q8 em todos os ambientes. Entre os ambientes testados temos variações nos resultados, sendo que a aplicação Q5 foi com melhor speed-up no físico com 17,09 pts, Azure com 15,82 pts e XenServer com 13,09 pts; o Q7 foi melhor em ambiente Azure com 9,09 pts, físico com 8,41 e XenServer com 7,59 pts; já o Q8 foi melhor em ambiente no XenServer com 2,56 pts, físico com 2,30 pts e Azure com 2,03 pts. Sendo que ao analisarmos uma média geral de speed-up das aplicações, temos o físico com 9,56 pts, o Azure com 8,98 pts, e o XenServer com 7,74 pts.

Para as aplicações da suíte NPB-NAS, houveram dois comportamentos similares, para o físico e o XenServer. Como o hardware em que as aplicações foram executadas é mesmo nos dois ambientes, os valores obtidos speed-up também foram próximos. A pequena diferença se deve a camada de virtualização. No ambiente físico foram obtidos os melhores valores de speed-up em todas as aplicações.

Já no ambiente Azure rodando as aplicações NPB-NAS obtivemos um comportamento diferente dos ambientes XenServer e físico. Algumas aplicações obtiveram melhor speed-up no Azure, já outra foi melhor no físico. Dentre as aplicações que foram melhor no Azure, temos a MG com 9,06 pts contra 2,30 pts; a FT com 8,44 pts contra 4,14 pts; e o CG, com 3,09 pts contra 1,05 pts. Já a única aplicação que obteve um melhor valor de speed-up no físico, temos a IS, com 4,14 pts, contra 3,02 pts. A Figura 7 apresenta os resultados para a suíte NPB-NAS. Podemos analisar que as aplicações MG, FT e CG tiveram os melhores resultados de speed-up no ambiente Azure. 


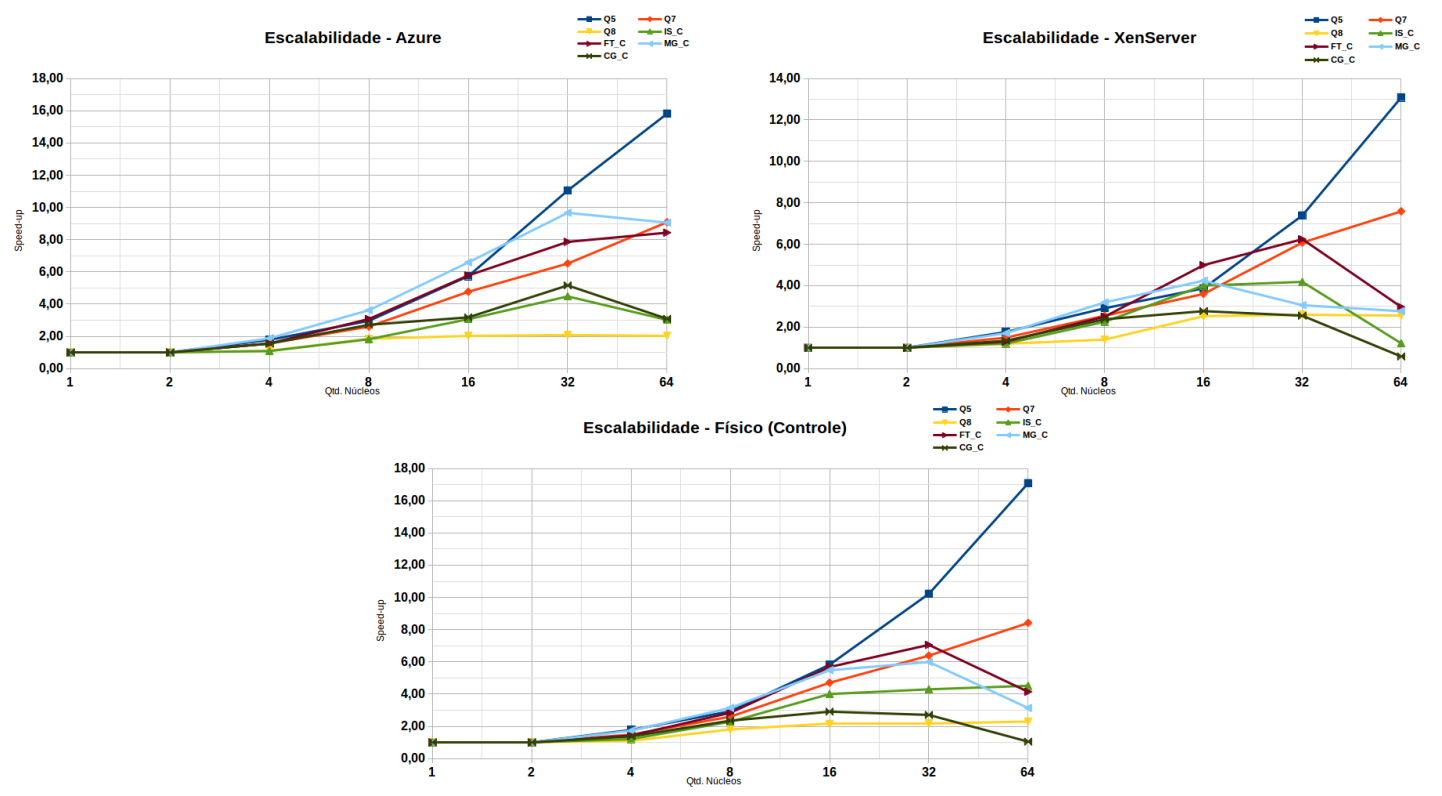

Figura 6. Escalabilidade das aplicações nos ambientes Público, Privado e Físico.
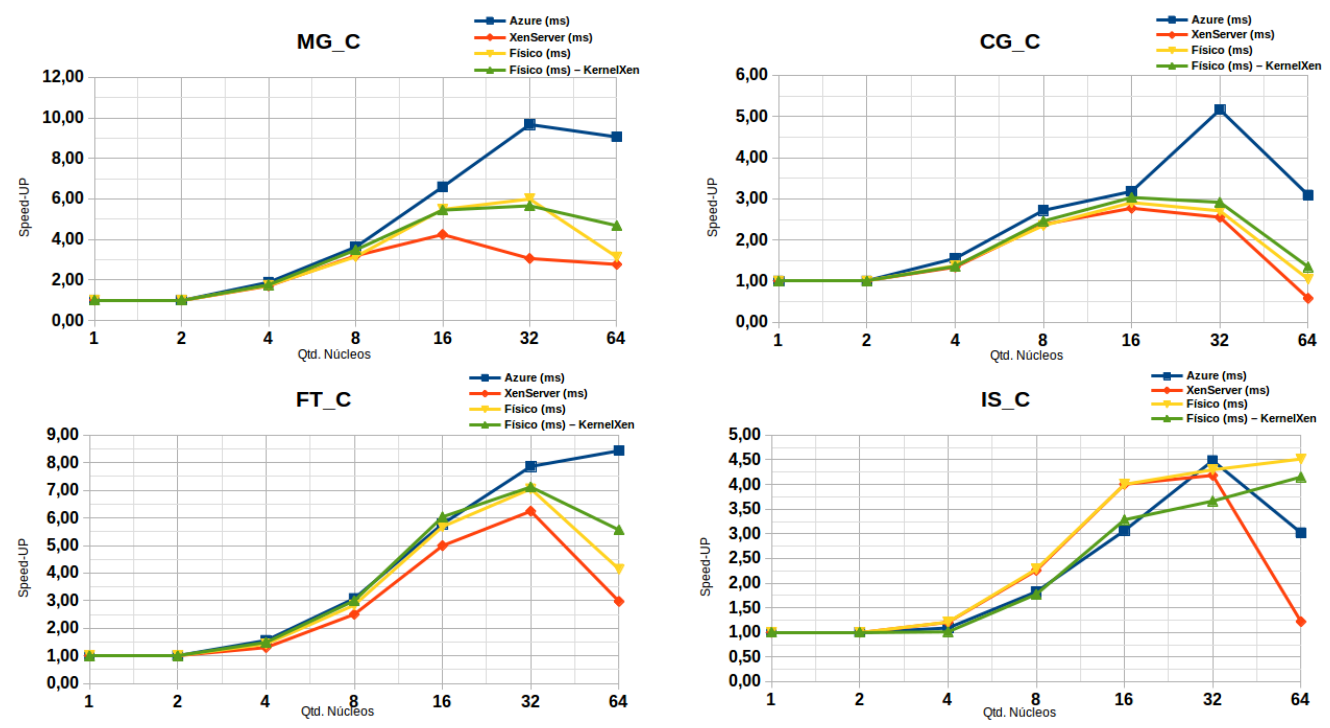

Figura 7. Avaliação da aplicação MG, CG, FT e IS.

Na Figura 8, temos as aplicações da suíte DGEO. Podemos averiguar que cluster físico obteve um melhor valor de speed-up no Q5, enquanto a nuvem pública Azure foi com uma leve vantagem no speed-up Q7, e a nuvem privada XenServer obteve melhores valores de speed-up no Q8. Ao analisarmos a nuvem pública com o físico, temos que o físico obteve os melhores valores de speed-up nas aplicações Q5 e Q8, sendo que no Q7 a diferença é muito pouca entre os dois ambientes, tendo o Azure obtido 9,09 pts e o físico 8,41 pts. A aplicação Q5 tem como padrão de desenvolvimento a característica de obter valores de speed-up próximos a $\frac{1}{2}$, enquanto a aplicação Q7, de ser $\frac{1}{4}$, e por sua vez a aplicação Q8 possui valor de speed-up próximo a nulo. 

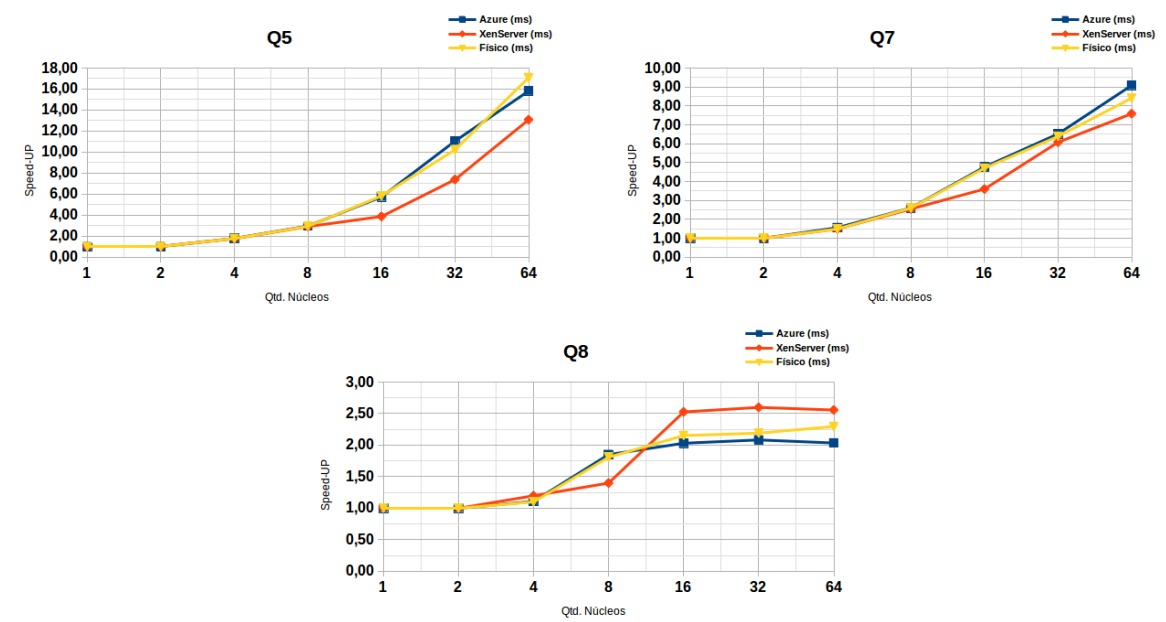

Figura 8. Avaliação das aplicação Q5, Q7 e Q8.

\section{Conclusão}

Este trabalho avaliou a escalabilidade de aplicativos de benchmark da suíte NPB-NAS, e de aplicações reais de processamentos de dados espaciais, a suíte DGEO, em uma nuvem pública, Azure, e uma nuvem privada, com o XenServer, usando como referência e controle um cluster de máquinas físicas sem virtualização de configuração similar a nuvem pública.

As aplicações foram escolhidas para demonstrar diferentes tipos de uso de recursos computacionais: aplicações CPU-bound e network-bound com diferentes níveis de speed-up. O intuito foi descobrir se, e como, a plataforma em nuvem compromete a execução de testes científicos de escalabilidade na nuvem.

Nos experimentos, o speed-up das aplicações quando executadas na nuvem pública foi até três vezes menor para aplicações $C P U$-bound, em comparação com um cluster físico com hardware equivalente. Apesar da qualidade superior da infraestrutura na nuvem pública, as aplicações também apresentaram tempo de execução até três vezes maior, comparando com o tempo de execução no cluster físico. Comparando o speed-up das aplicações entre as nuvens pública e privada foi verificado que o mesmo não é afetado somente pela camada adicional de virtualização, mas também por outros fatores da plataforma de nuvem, como o uso de máquinas vizinhas.

Os resultados alcançados indicam que apesar de conveniente, testar a escalabilidade de aplicações distribuídas em plataformas virtuais (nuvem pública ou privada) pode levar a interpretações incorretas ou imprecisas a respeito da escalabilidade de alguns tipos de aplicações distribuídas.

Um esforço adicional é necessário em trabalhos futuros para generalizar ou expandir os resultados para outras plataformas de nuvens públicas e privadas.

\section{Referências}

ABRAMSON, D. et al. Intel Virtualization Technology for Directed I/O. White Paper, Intel: $\quad$ https://www-ssl.intel.com/content/dam/www/public/us/en/documents/productspecifications/vt-directed-io-spec.pdf, n. 3, 2014. 
AMD. AMD I/O Virtualization Technology (IOMMU) Specification. White Paper, AMD: http://developer.amd.com/wordpress/media/2012/10/488821.pdf, v. 2.00, 2011.

BAILEY, D. H. et al. The nas parallel benchmarks. International Journal of High Performance Computing Applications, SAGE Publications, v. 5, n. 3, p. 63-73, 1991.

BAKHSHAYESHI, R.; AKBARI, M. K.; JAVAN, M. S. Performance Analysis of Virtualized Environments Using HPC Challenge Benchmark Suite and Analytic Hierarchy Process. In: Intelligent Systems (ICIS), 2014 Iranian Conference on. Higher Education Complex of Bam, Highway Persian Gulf, Bam, Iran: [s.n.], 2014. p. 1-6.

BARKER, S. K.; SHENOY, P. Empirical Evaluation of Latency-sensitive Application Performance in the Cloud. In: Proceedings of the First Annual ACM SIGMM Conference on Multimedia Systems. New York, NY, USA: ACM, 2010. p. 35-46.

EXPÓSITO, R. R. et al. Performance analysis of hpc applications in the cloud. Future Generation Computer Systems, Elsevier, v. 29, n. 1, p. 218-229, 2013.

FERREIRA, C. H. et al. Identificação de Gargalos de Desempenho em Ambientes Virtuais para uso em Computação em Nuvem. XIII Workshop em Desempenho de Sistemas Computacionais e de Comunicação, Braília - DF, Brasil, p. 1 - 14, 2014.

HILL, Z. et al. Early Observations on the Performance of Windows Azure. In: Proceedings of the 19th ACM International Symposium on High Performance Distributed Computing. New York, NY, USA: ACM, 2010. (HPDC '10), p. 367-376.

HUBER, N. et al. Evaluating and modeling virtualization performance overhead for cloud environments. In: CLOSER. [S.1.: s.n.], 2011. p. 563-573.

MEI, Y. et al. Performance Analysis of Network I/O Workloads in Virtualized Data Centers. Services Computing, IEEE Transactions on, IEEE Transactions on Services Computing, p. 48-63, 2013.

OLIVEIRA, T. B. de; COSTA, F. M.; RODRIGUES, V. J. S. Definição de Planos de Execução Distribuídos para Consultas de Junção Espacial usando Histogramas Multidimensionais. In: Proc. of XXX SBBD. Petrópolis: [s.n.], 2015. p. 89-100.

OLIVEIRA, T. B. de et al. Avaliação Comparativa de Escalabilidade de Aplicações de Alto Desempenho em Cluster Físico e na Nuvem. In: Anais do WPerformance - CSBC 2015. Recife: [s.n.], 2015. p. 1-12.

SIVATHANU, S.; DONG, X. Performance analysis of network i/o workloads in virtualized data centers. IEEE Transactions on Services Computing, p. 48-63, 2013.

TUDORAN, R. et al. A Performance Evaluation of Azure and Nimbus Clouds for Scientific Applications. In: Proceedings of the 2Nd International Workshop on Cloud Computing Platforms. New York, NY, USA: ACM, 2012. p. 4:1-4:6.

WALTERS, J. P. et al. A comparison of virtualization technologies for hpc. In: IEEE. 22nd International Conference on Advanced Information Networking and Applications (aina 2008). [S.1.], 2008. p. 861-868.

YOUNGE, A. J. et al. Analysis of virtualization technologies for high performance computing environments. In: IEEE. Cloud Computing (CLOUD), 2011 IEEE International Conference on. [S.1.], 2011. p. 9-16. 